\title{
On strongly non-linear systems discretization
}

\author{
David Panek \\ Department of Theory of Electrical Engineering \\ Faculty of Electrical Engineering, University of West Bohemia \\ Czech Republic , 32600 Pilsen \\ Email: panek50@kte.zcu.cz
}

\begin{abstract}
This paper deals with the discretization of strongly non-linear systems. Proposed method is based on application of so called dissipation normal form (discrete and continuoustime). The paper shows that trough the discretization it is possible to conserve chaotic behaviour of the system. During the discretization the chaotic behaviour is usually considered as critically sensitive to changes in initial conditions, parameters and inputs. It is interesting that some aspects of the chaotic behaviour are conserved even for systems with quantized values of states and parameters.
\end{abstract}

\section{INTRODUCTION}

Non-linear systems with chaotic behaviour (see the exact definition bellow) represent a fast-growing sector of nonlinear dynamical systems theory. To have an opportunity to build discrete-time models of examined objects or phenomena would be useful in a large number of applications. There is no doubt that the digital signal processing offers much wider possibilities than the continuous processing. Discrete implementation of originally continuous-time systems can be used for pseudo-random sequences generation, for the synchronisation between a transceiver and a receiver in special communications schemes or for any purposes requiring deterministic signals with certain degree of irregularity. The main goal of the paper is to propose the methodology which can be used for the discretization of strongly non-linear systems with chaotic behaviour.

\section{Discretization METhod CONSERVING QUALITATIVE BEHAVIOUR OF THE SYSTEM}

Suppose a system desribed by the dissipation normal form [3]. It is possible to divide the matrix of dynamics $\boldsymbol{A}$ into a sum of particular matrices

$$
\begin{aligned}
& \boldsymbol{A}=\left[\begin{array}{cccc}
-\alpha_{1} & 0 & \ldots & 0 \\
0 & 0 & \ldots & 0 \\
\vdots & \vdots & \ddots & \vdots \\
0 & 0 & \ldots & 0
\end{array}\right]+\left[\begin{array}{cccc}
0 & -\alpha_{2} & \ldots & 0 \\
\alpha_{2} & 0 & \ldots & 0 \\
\vdots & \vdots & \ddots & \vdots \\
0 & 0 & \ldots & 0
\end{array}\right] \ldots \\
&+ {\left[\begin{array}{cccc}
0 & \ldots & 0 & 0 \\
\vdots & \vdots & \ddots & \vdots \\
0 & \ldots & 0 & -\alpha_{n} \\
0 & \ldots & \alpha_{n} & 0
\end{array}\right], } \\
& \boldsymbol{A}=\sum_{i=1}^{n} \boldsymbol{A}_{i} .
\end{aligned}
$$

Then it is possible to apply a standard method of discretization on the matrix sum in a form

$$
\hat{\boldsymbol{A}}=\exp \left(\sum_{i=1}^{n} \boldsymbol{A}_{i} T_{\mathrm{s}}\right)=\prod_{i=1}^{n} \exp \left(\boldsymbol{A}_{i} T_{\mathrm{s}}\right)=\hat{\boldsymbol{A}}_{1} \cdot \hat{\boldsymbol{A}}_{2} \cdots \hat{\boldsymbol{A}}_{n}
$$

where

$$
\begin{gathered}
\hat{\boldsymbol{A}}_{1}=\left[\begin{array}{cccc}
\exp \left(-\alpha_{1} T_{\mathrm{s}}\right) & 0 & \ldots & 0 \\
0 & 1 & \ldots & 0 \\
\vdots & \vdots & \ddots & \vdots \\
0 & 0 & \ldots & 1
\end{array}\right], \\
\hat{\boldsymbol{A}}_{i}=\left[\begin{array}{cccccc}
1 & 0 & 0 & \ldots & 0 & 0 \\
0 & 1 & 0 & \ldots & 0 & 0 \\
\vdots & \vdots & \vdots & \ddots & \vdots & \vdots \\
0 & \ldots & \cos \left(\alpha_{i} T_{\mathrm{s}}\right) & -\sin \left(\alpha_{i} T_{\mathrm{s}}\right) & \ldots & 0 \\
0 & \ldots & \sin \left(\alpha_{i} T_{\mathrm{s}}\right) & \cos \left(\alpha_{i} T_{\mathrm{s}}\right) & \ldots & 0 \\
\vdots & \vdots & \vdots & \ddots & \vdots & \vdots \\
0 & 0 & 0 & \ldots & 1 & 0 \\
0 & 0 & 0 & \ldots & 0 & 1
\end{array}\right] .
\end{gathered}
$$

It can be proven that using this method of discretization we can get directly the discrete-time dissipation normal form. The dissipation normal form is closely related to the lattice-ladder structure of linear time-invariant discrete-time filters [2].

The important point is that the mentioned method conserves the qualitative behaviour of the system. If we suppose the abstract energy function in a form

$$
E=\frac{1}{2} \sum_{i=1}^{n} x_{i}^{2}=\frac{1}{2} \boldsymbol{x}^{\mathrm{T}} \boldsymbol{x}
$$

then the evolution of energy can be described by the expression

$$
\begin{gathered}
\frac{\mathrm{d} E}{\mathrm{~d} t}=\frac{1}{2}\left(\dot{\boldsymbol{x}}^{\mathrm{T}} \boldsymbol{x}+\boldsymbol{x}^{\mathrm{T}} \dot{\boldsymbol{x}}\right)= \\
=\frac{1}{2}\left[\boldsymbol{x}^{\mathrm{T}} \boldsymbol{A}^{\mathrm{T}}(\boldsymbol{x}) \boldsymbol{x}+\boldsymbol{x}^{\mathrm{T}} \boldsymbol{A}(\boldsymbol{x}) \boldsymbol{x}\right]=-\alpha_{1}(\boldsymbol{x}) x_{1}^{2} .
\end{gathered}
$$

The expression above can be interpreted in the sense that on intervals where the function $\alpha_{1}(\boldsymbol{x})>0$ is the system antidissipative, on intervals where $\alpha_{1}(\boldsymbol{x})<0$ is the system dissipative and for $\alpha_{1}(\boldsymbol{x})=0$ is the system conservative. For a discrete-time system it is possible to write the energy conservation law in a form

$$
\Delta E(k)=E(k+1)-E(k)=
$$




$$
\begin{gathered}
=\frac{1}{2}\left[\boldsymbol{x}^{T}(k+1) \boldsymbol{x}(k+1)-\boldsymbol{x}^{T}(k) \boldsymbol{x}(k)\right]= \\
=\frac{1}{2} \boldsymbol{x}^{T}(k)\left[\hat{\boldsymbol{A}}^{\mathrm{T}} \hat{\boldsymbol{A}}-\mathbf{I}\right] \boldsymbol{x}(k)
\end{gathered}
$$

We can see that for all $\boldsymbol{A}_{i}, i \neq 1$ is the result of the product $\boldsymbol{A}_{i}^{\mathrm{T}} \boldsymbol{A}_{i}$ a unit matrix and the energy change can be expressed by the equation

$$
\Delta E(k)=\frac{1}{2}\left(\Delta_{1}-1\right) x_{1}^{2}=\frac{1}{2}\left(e^{-\alpha_{1} T_{s}}-1\right) x_{1}^{2},
$$

this means that there is exactly the same condition for the dissipativity of the discretised system as for the continuoustime system.

\section{EXAMPLE}

Let us have a strongly non-linear system representation by equations

$$
\begin{aligned}
\mathcal{R}\{S\}: & \dot{x}_{1}=x_{2}+x_{3}, \\
\dot{x}_{2} & =-x_{1}+0.5 x_{2}, \\
\dot{x}_{3} & =x_{1}^{2}-x_{3} \\
y & =x_{1}
\end{aligned}
$$

It is not possible to use the discretization method mentioned above for this system representation. If the discretization is provided directly, the result is a non-stable discrete system. Our first task is to find a representation in the form specified by. An equivalent system representation is given by equations

$$
\begin{aligned}
\mathcal{R}\{\bar{S}\}: & \dot{\bar{x}}_{1}=-\alpha_{1} \bar{x}_{1}+\alpha_{2} \bar{x}_{2}, \\
\dot{\bar{x}}_{2} & =\bar{x}_{1}+\bar{x}_{3}, \\
\dot{\bar{x}}_{3} & =-\alpha_{3} \bar{x}_{2}, \\
\bar{y} & =\bar{x}_{2},
\end{aligned}
$$

where $\alpha_{1}=0.5, \alpha_{2}=2 \bar{x}_{2}+1.5, \alpha_{3}=\bar{x}_{2}+2$. It is possible to find a diffeomorphism $\boldsymbol{T}(\boldsymbol{x})$ that transforms the representation $\mathcal{R}\{S\}$ to the representation $\mathcal{R}\{\bar{S}\}$ from the inner invariants equality requirement .

$$
\begin{aligned}
{\left[\begin{array}{c}
\mathcal{L}_{f}^{0}(h) \\
\mathcal{L}_{f}^{1}(h) \\
\mathcal{L}_{f}^{2}(h)
\end{array}\right] } & =\left[\begin{array}{c}
\mathcal{L}_{f}^{0}(\bar{h}) \\
\mathcal{L}_{f}^{1}(\bar{h}) \\
\mathcal{L}_{f}^{2}(\bar{h})
\end{array}\right] \\
{\left[\begin{array}{c}
x_{1} \\
x_{2}+x_{3} \\
-x_{1}+x_{1}^{2}+0.5 x_{2}-x_{3}
\end{array}\right] } & =\left[\begin{array}{c}
\bar{x}_{2} \\
\bar{x}_{1}+\bar{x}_{3} \\
-0.5 \bar{x}_{1}+\bar{x}_{2}^{2}-0.5 \bar{x}_{2}
\end{array}\right]
\end{aligned}
$$

After several manipulations we can obtain the transformation in a form

$$
\left[\begin{array}{l}
\bar{x}_{1} \\
\bar{x}_{2} \\
\bar{x}_{3}
\end{array}\right]=\left[\begin{array}{ccc}
1 & -1 & 2 \\
1 & 0 & 0 \\
-1 & 2 & 1
\end{array}\right]\left[\begin{array}{l}
x_{1} \\
x_{2} \\
x_{3}
\end{array}\right]
$$

The comparison between outputs $y(t)$ and $\bar{y}(t)$ of equivalent system representations is depicted at the Figure 1. However the outputs seem to be identical, on longer time interval the difference between outputs rapidly increases. The interval with negligible difference between outputs of both realizations

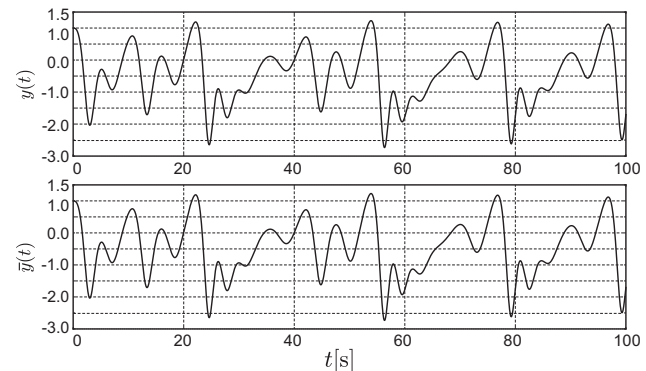

Fig. 1. Time evolution of chaotic continuous and discretized system outputs

depends on the method and parameters used for the numerical integration of given differential equations. The reason is that in different representations rounding errors influence results in a different way and the behaviour of the system is chaotic thus extremely sensitive to changes in initial conditions. Once the system is expressed by the representation (9), it is possible to provide the discretization in the standard way.

\section{CONCLUSIONS}

The main goal of the paper is to show that it is possible to find a discrete-time representation of strongly non-linear systems with chaotic behaviour. The important result is that the presented method of discretization conserves qualitative behaviour of the discretized system. The second important result is that it is possible to find an interval (which depends mainly on sampling frequency) with an acceptable difference between state variables values of the original continuous-time and the discretized system. In the paper [3] was shown that discrete-time non-linear systems can be modified to digital (discrete in time and level) systems that can generate pseudorandom sequences. Dicretization of continuous-time chaotic systems can lead to pseudo-random sequences generators with specific behaviour. The next important point is that for discrete-time systems new methods of synchronization based on digital signal processing can be proposed. The methodology of discretization of chaotic systems also gives a new perspective on the problem of reconstruction of trajectory in the states space (the most common method was presented in [1]).

\section{ACKNOWLEDGMENT}

This work was supported by European Regional Development Fund and Ministry of Education, Youth and Sports of the Czech Republic under the project No. CZ.1.05/2.1.00/03.0094 - RICE.

\section{REFERENCES}

[1] P. Grassberger and I. Procaccia. Characterization of strange attractors. Physical Review Letters, 5:346-349, 1983. ISBN: 80-7082-951-6.

[2] H. Kimura. Generalized schwarz form and Lattice-Ladder realizations of digital filters. IEEE Transactions on Circuit and Systems, 32(11):11301139, November 1985. ISSN: 0098-4094.

[3] D. Pánek, J. Lahoda, J. Hrušák, and M. Štork. On chaotic systems synthesis and synchronization. Acta Technica CSAV (Ceskoslovensk Akademie Ved), 54(2):179-198, 2009. 